\title{
Optical spectroscopy of X-ray sources in the Taurus molecular cloud: discovery of ten new pre-main sequence stars ${ }^{\star}$
}

\author{
L. Scelsi ${ }^{1}$, G. Sacco ${ }^{3,1}$, L. Affer ${ }^{1,2}$, C. Argiroffi ${ }^{2}$, I. Pillitteri ${ }^{2}$, A. Maggio ${ }^{1}$, and G. Micela ${ }^{1}$ \\ 1 INAF - Osservatorio Astronomico di Palermo, Piazza del Parlamento 1, 90134 Palermo, Italy \\ e-mail: scelsi@astropa.unipa.it \\ 2 Dipartimento di Scienze Fisiche ed Astronomiche, Sezione di Astronomia, Università di Palermo, Piazza del Parlamento 1, \\ 90134 Palermo, Italy \\ ${ }^{3}$ Consorzio COMETA, via S. Sofia 64, 95123 Catania, Italy
}

Received 12 May 2008 / Accepted 4 August 2008

\section{ABSTRACT}

\begin{abstract}
Aims. We have analyzed optical spectra of $25 \mathrm{X}$-ray sources identified as potential new members of the Taurus molecular cloud (TMC), in order to confirm their membership in this star-forming region.

Methods. Fifty-seven candidate members were previously selected among the X-ray sources in the XEST survey, having a 2MASS counterpart compatible with a pre-main sequence star based on color-magnitude and color-color diagrams. We obtained highresolution optical spectra for 7 of these candidates with the SARG spectrograph at the TNG telescope, which were used to search for lithium absorption and to measure the $\mathrm{H} \alpha$ line and the radial and rotational velocities. Then, 18 low-resolution optical spectra obtained with the instrument DOLORES for other candidate members were used for spectral classification, for $\mathrm{H} \alpha$ measurements, and to assess membership together with IR color-color and color-magnitude diagrams and additional information from the X-ray data.

Results. We found that 3 sources show lithium absorption, with equivalent widths ( $E W \mathrm{~s})$ of $\sim 500 \mathrm{~m} \AA$, broad spectral line profiles, indicating rotational velocities of $\sim 20-40 \mathrm{~km} \mathrm{~s}^{-1}$, radial velocities consistent with those for known members, and H $\alpha$ emission. Two of them are classified as new weak-lined T Tauri stars, while the $E W(\sim-9 \AA)$ of the $\mathrm{H} \alpha$ line and its broad asymmetric profile clearly indicate that the third star (XEST-26-062) is a classical T Tauri star. Fourteen sources observed with DOLORES are M-type stars. Fifteen sources show $\mathrm{H} \alpha$ emission. Six of them have spectra that indicate surface gravity lower than in main sequence stars, and their de-reddened positions in IR color-magnitude diagrams are consistent with their derived spectral type and with pre-main sequence models at the distance of the TMC. The K-type star XEST-11-078 is confirmed as a new member on the basis of the strength of the $\mathrm{H} \alpha$ emission line. Overall, we confirm membership to the TMC for 10 out of $25 \mathrm{X}$-ray sources observed in the optical. Three sources remain uncertain.
\end{abstract}

Key words. techniques: spectroscopic - stars: luminosity function, mass function - stars: pre-main sequence Galaxy: open clusters and associations: individual: Taurus molecular cloud

\section{Introduction}

The formation of stars and planets and the evolution of stellar properties during the early stages of their lives (internal structure, angular momentum, magnetic activity, and X-ray emission in low-mass stars) is one of the most intriguing problems in astrophysics. The fragmentation of large clouds and the subsequent gravitational collapse of molecular cores is a stochastic process that leads to a population of stellar and sub-stellar objects with a variety of masses. The details of these physical mechanisms are still not clear; turbulence and density fluctuations appear to be crucial (Klessen 2001; Goodwin et al. 2004), but other factors probably play important roles, such as the presence of magnetic fields (Padoan \& Nordlund 1999) or the shock waves propagating from supernovae explosions that may determine the fragmentation of clouds (Truelove 1997).

Theoretical models of cloud fragmentation and core collapse should reproduce the initial mass function (IMF) of a

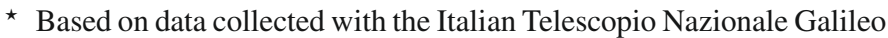
(TNG) operated on the island of La Palma by the Centro Galileo Galilei of INAF (Istituto Nazionale di Astrofisica) at the Spanish Observatorio del Roque del los Muchachos of the Instituto de Astrofisica de Canarias.
}

star-forming region (SFR), that is the mass distribution of a stellar group just born. Significant differences between the IMFs derived for the Taurus-Auriga SFR (Luhman et al. 2003a), which hosts a distributed mode of star formation, and for much denser regions containing massive stars, such as Orion and IC 348 (Muench et al. 2002; Luhman et al. 2003b, respectively), have suggested that the star formation process may be affected by the physical conditions of the environment where stars form. However, previous studies of the TMC may not be complete at low masses, while the most recent surveys (Briceño et al. 2002; Luhman et al. 2003a; Luhman 2004; Guieu et al. 2006; Luhman et al. 2006) have allowed discovery of new low-mass stars and brown dwarfs, but were focused on limited portions of the region and are not spatially complete. In fact, studies of the TMC are also complicated by its large extension in the sky ( 100 square degrees, also due to its distance of $140 \mathrm{pc}$ ), and require long observational campaigns with sufficiently deep exposures. More complete studies of the Taurus population are therefore needed to assess the IMF shape with greater confidence, especially at the very low-mass end.

We started a search for new members of the Taurus-Auriga SFR (Scelsi et al. 2007), based on X-ray data from the 
XMM-Newton Extended Survey of the Taurus Molecular Cloud (XEST, Güdel et al. 2007) and on near-infrared data from the 2MASS point source catalog (Skrutskie et al. 2006). This is a different approach with respect to the above mentioned searches for new Taurus members, where candidates were selected based on optical and IR data. Since intense X-ray emission (10 to $10^{4}$ times the solar level, see, e.g., Feigelson \& Montmerle 1999; Stelzer \& Neuhäuser 2001; Ozawa et al. 2005) is a ubiquitous characteristic of pre-main sequence solar-type stars and thanks to the relatively low interstellar absorption at these wavelengths, X-ray observations are particularly efficient at detecting the population of young objects, including very low-mass stars that are faint in the optical bands, and can therefore serve as a complement to optical/IR searches. Moreover, since non-accreting "weak-lined" T Tauri stars (WTTSs) are generally more luminous in X rays (e.g. Briggs et al. 2007) and less absorbed than classical T Tauri stars (CTTSs), which are still surrounded by a thick circumstellar accretion disk, the former are expected to be selected more efficiently in X-ray surveys. This is particularly important, because it helps to reduce possible biases introduced by optical/IR surveys, which may favour the detection of CTTSs owing to their strong $\mathrm{H} \alpha$ emission and IR excess, and hence allows us to properly address fundamental issues such as the estimate of disk lifetimes, with important consequences on our understanding of the evolution of the angular momentum during the earlier phases of stellar life and in the formation of planetary systems.

In this paper we present the results of the analysis of followup optical spectroscopy obtained at the Telescopio Nazionale Galileo (TNG) for 25 out of 57 candidate Taurus members selected in the previous work by Scelsi et al. (2007). The paper is organized as follows. Section 2 summarizes the main information about the XEST survey and the X-ray/IR based selection of potential new members; in Sect. 3 we describe the followup TNG observations of the optically brightest candidates. In Sect. 4 we present the results of the optical spectroscopy, while in Sect. 5 we determine the stellar properties. Finally, we discuss our results in Sect. 6.

\section{X-ray/IR selection of new TMC candidates}

Scelsi et al. (2007) searched for TMC candidate members among the X-ray sources detected in the fields of the XEST survey (Güdel et al. 2007). This large project consists of 27 XMM-Newton observations of the Taurus molecular cloud covering the densest concentrations of molecular gas in this region, as traced by $\mathrm{CO}$ emission. The exposure times are typically $\sim 30 \mathrm{ks}$ each, although a few fields have longer exposures (in the range $\sim 45-120 \mathrm{ks}$ ), while others were severely contaminated by a high level of background radiation that reduced the useful exposure time significantly. The total surveyed area covers about 5 square degrees of the TMC and contains $\sim 150$ members, that is, about half its known total population.

Candidate members were selected among the X-ray sources with an IR counterpart in the 2MASS catalog and not previously identified with known objects. The X-ray sources without an IR counterpart were not considered, since their number and global X-ray properties are consistent with their nature likely being an extragalactic one, although some heavily absorbed Taurus members might be found among them. We refer the reader to Scelsi et al. (2007) for details about the selection procedure, based on the consistency of the 2MASS counterparts with PMS isochrones by Baraffe et al. (1998) and Siess et al. (2000), calculated for the distance of the TMC (140 pc), in
IR color-color ( $J-H$ vs. $H-K$ ) and color-magnitude ( $J$ vs. $J-K$ and $H$ vs. $H-K)$ diagrams. Among the 57 candidates selected in this way, we identified 12 candidates with a higher probability of membership based on their X-ray properties, namely high plasma temperatures and/or presence of strong flares in their light curves, both characteristic of young stars.

The candidate member XEST-08-003 was also identified as a potential TMC member by Jones \& Herbig (1979), who found its proper motion consistent with the average of the Taurus members, while XEST-26-062 was noticed by Strom et al. (1976) as an IR source in the cloud L1517.

\section{Optical sample and observations}

We chose the candidates to be observed at the TNG after searching for optical counterparts in the USNO catalog. We selected 38 sources with magnitude in the $R$ band brighter than 17.5, which could be observed in a reasonable amount of time (4 nights in total) with relatively high ( $~ 50-80)$ signal-to-noise ratios. However, bad weather and seeing conditions during some nights restricted the observed stars to 27 . The 8 brightest stars $(R \simeq 10.2-13.4)$ were observed at high spectral resolution with SARG, while the remaining 19 stars $(R \simeq 13.8-17.5)$ at low resolution with DOLORES/LRS. The SARG spectrum of XEST-06-045 and the DOLORES spectrum of XEST-26-135 turned out to be too weak and were not analyzed. The 25 sample stars considered in this work are listed in Table 1, together with relevant information, while Fig. 1 shows their positions in the $J-H$ vs. $H-K$ and $J$ vs. $J-K$ diagrams.

The observations of the candidates listed in Table 1 were performed on the nights of 2007 January 20-23 with the instruments on the $3.6 \mathrm{~m}$ Telescopio Nazionale Galileo at the La Roque de los Muchachos Observatory. The high-resolution spectrograph SARG was operated with the $61 \AA \mathrm{mm}^{-1} \mathrm{CD} 3$ grism $\left(\lambda_{\text {blaze }}=5890 \AA\right)$, which produced wavelength coverage from 4620 to $7920 \AA$, with a yellow filter and $1.6^{\prime \prime}$ slit providing spectral resolution $R \sim 29000$. Exposure times ranged from 900 up to $5400 \mathrm{~s}$ for the 3 faintest sources observed with this instrument. Data reduction of these echelle spectra (bias subtraction, flat fielding, extraction of background-subtracted spectra, and wavelength calibration) was performed with the tasks within the IRAF ${ }^{1}$ software system. The low-resolution spectra were obtained with DOLORES (LRS) configured in long-slit spectroscopy mode; we used the VHR-V grism and 1 " slit. This setup provides wavelength coverage from 4650 to $6800 \AA$ and spectral resolution of $3.8 \AA(R \sim 1500)$. Exposure times ranged from 600 to $5400 \mathrm{~s}$. The LRS spectra were reduced using both IRAF tasks and procedures written in IDL. These spectra were not corrected for the instrumental response and airmass.

\section{Results}

\subsection{High-resolution spectra}

The SARG spectra of seven candidates (see Table 1) were used to measure the strength of $\mathrm{Li}$ absorption and $\mathrm{H} \alpha$ line (Fig. 2) and to measure radial and rotational velocities. We also performed spectral classification. Typical signal-to-noise $(S / N)$ ratios of the SARG spectra are in the range 60-80 for all stars, with

\footnotetext{
1 IRAF (Image Reduction and Analysis Facility) is distributed by the National Optical Astronomy Observatories, operated by the Association of Universities for Research in Astronomy, Inc., under cooperative agreement with the National Science Foundation.
} 
Table 1. List of TMC candidates observed at the TNG.

\begin{tabular}{|c|c|c|c|c|c|c|c|c|c|c|}
\hline $\mathrm{XEST} \mathrm{id}^{a}$ & $\begin{array}{c}\mathrm{RA}^{b} \\
\mathrm{~h} \mathrm{~m} \mathrm{~s}\end{array}$ & $\begin{array}{c}\operatorname{Dec}^{b} \\
\operatorname{deg}{ }^{\prime} \quad "\end{array}$ & $\begin{array}{l}\text { X-ray } \mathrm{CR}^{c} \\
\text { cts ks }\end{array}$ & $\operatorname{var}^{d}$ & desig. 2MASS & $\begin{array}{c}R^{e} \\
\mathrm{mag}\end{array}$ & $\begin{array}{c}J^{f} \\
\mathrm{mag}\end{array}$ & $\begin{array}{c}H^{f} \\
\mathrm{mag}\end{array}$ & $\begin{array}{c}K^{f} \\
\mathrm{mag}\end{array}$ & instr. \\
\hline $27-022$ & 35337.30 & +32458.3 & $7.9 \pm 0.8$ & & $03533730+3204582$ & 10.2 & 8.38 & 7.91 & 7.78 & SARG \\
\hline $21-059$ & 42225.60 & +281233.4 & $8.9 \pm 0.8$ & & $04222559+2812332$ & 10.8 & 9.26 & 8.75 & 8.62 & SARG \\
\hline $11-035$ & 42143.73 & +264722.6 & $2.1 \pm 0.5$ & & $04214372+2647225$ & 11.1 & 9.61 & 8.98 & 8.83 & SARG \\
\hline 09-042 & 43558.98 & +223834.8 & $269 \pm 3$ & $\mathrm{y}$ & $04355892+2238353$ & 11.3 & 9.32 & 8.60 & 8.37 & SARG \\
\hline 04-060 & 43355.61 & +24251.9 & $1.3 \pm 0.3$ & & $04335562+2425016$ & 12.8 & 10.06 & 9.29 & 9.00 & SARG \\
\hline $26-062$ & 45555.99 & +303621.9 & $9.5 \pm 0.4$ & $\mathrm{y}$ & $04555605+3036209$ & 13.4 & 10.47 & 9.66 & 9.27 & SARG \\
\hline $08-003$ & 43456.91 & +225835.9 & $331 \pm 6^{g}$ & $\mathrm{y}$ & $04345693+2258358$ & 13.4 & 10.47 & 9.59 & 9.27 & SARG \\
\hline $18-059$ & 43433.21 & +26240.7 & $6.7 \pm 1.1$ & & $2+2602403$ & 13.8 & 11.18 & 10.54 & 10.28 & LRS \\
\hline $16-045$ & 42039.19 & +271732.1 & $30.4 \pm 2.4$ & & $8+2717317$ & 14.4 & 10.50 & 9.86 & 9.56 & LRS \\
\hline 20-071 & 41452.31 & +2860.3 & $159 \pm 3$ & & $04145234+2805598$ & 14.4 & 9.53 & 8.21 & 7.71 & LRS \\
\hline $06-041$ & 40424.48 & +2611 12.1 & $17.1 \pm 1.1$ & & $04042449+2611119$ & 14.7 & 12.10 & 11.45 & 11.19 & LRS \\
\hline 17-059 & 43352.50 & +225627.9 & $57.4 \pm 1.9$ & & $04335252+2256269$ & 14.7 & 10.24 & 9.47 & 9.11 & LRS \\
\hline 08-014 & 43513.18 & +22 5920.5 & $62.9 \pm 1.9$ & $\mathrm{y}$ & $04351316+2259205$ & 14.9 & 11.13 & 10.53 & 10.25 & LRS \\
\hline 15-034 & 42936.24 & +263423.5 & $24.4 \pm 1.0$ & $\mathrm{y}$ & $04293623+2634238$ & 15.0 & 11.56 & 10.94 & 10.65 & LRS \\
\hline $05-027$ & 4403.62 & +255354.9 & $109 \pm 5$ & & $04400363+2553547$ & 15.2 & 9.81 & 8.48 & 7.91 & LRS \\
\hline $15-075$ & 43017.02 & +262226.5 & $24.6 \pm 2.5$ & & $04301702+2622264$ & 15.5 & 11.80 & 11.16 & 10.91 & LRS \\
\hline 08-049 & 43552.87 & +225058.5 & $118 \pm 2$ & $\mathrm{y}$ & $04355286+2250585$ & 15.5 & 10.99 & 10.11 & 9.75 & LRS \\
\hline 08-047 & 43552.12 & +22553.6 & $5.8 \pm 0.6$ & & $04355209+2255039$ & 15.7 & 11.31 & 10.23 & 9.81 & LRS \\
\hline $11-087$ & 42224.03 & +264626.3 & $13.4 \pm 1.1$ & & $04222404+2646258$ & 15.8 & 11.09 & 10.19 & 9.77 & LRS \\
\hline $19-002$ & 43146.35 & +25 5840.6 & $3.1 \pm 0.9$ & & 58404 & 16.0 & 13.33 & 12.62 & 12.31 & LRS \\
\hline 27-084 & 35410.61 & +314858.2 & $18.0 \pm 0.7^{g}$ & $\mathrm{y}$ & $03541064+3148573$ & 16.1 & 12.39 & 11.64 & 11.36 & LRS \\
\hline 08-033 & 43542.03 & +225222.5 & $27.2 \pm 0.9$ & & $04354203+2252226$ & 16.3 & 11.25 & 10.39 & 9.99 & LRS \\
\hline $22-111$ & 43226.88 & +18 1823.1 & $9.9 \pm 1.3$ & $\mathrm{y}$ & $04322689+1818230$ & 16.4 & 12.80 & 12.00 & 11.69 & LRS \\
\hline $11-078$ & 42215.68 & +26576.4 & $9.4 \pm 0.5$ & $\mathrm{y}$ & $04221568+2657060$ & 17.1 & 13.81 & 12.62 & 12.03 & LRS \\
\hline $12-012$ & 43451.64 & +24443.0 & $3.3 \pm 0.8$ & & $04345164+2404426$ & 17.5 & 13.19 & 12.37 & 11.97 & LRS \\
\hline
\end{tabular}

Note: sources in bold face have a higher probability of membership on the basis of X-ray light curve and/or spectral analysis (see Scelsi et al. 2007). ${ }^{a}$ Name of the X-ray source in the XEST catalog (the first 2 digits mark the field of the survey); ${ }^{b}$ coordinates of the optical counterpart in the USNO catalog; ${ }^{c}$ equivalent on-axis count-rate for the PN in the $0.5-7.3 \mathrm{keV}$ band, averaged over the entire observation; ${ }^{d} \mathrm{X}$-ray source variable according to the maximum likelihood algorithm described in Stelzer et al. (2007); ${ }^{e}$ magnitude in the $R$ band reported in the USNO catalog; ${ }^{f}$ infrared magnitudes reported in the 2MASS catalog; ${ }^{g}$ quiescent count-rates (cts ks ${ }^{-1}$ ) for sources with strong flares: $180 \pm 40$ (XEST-08-003), $15.0 \pm 4.3($ XEST-27-084).

the exception of XEST-08-003 $(S / N \sim 45)$ and XEST-26-062 $(S / N \sim 35)$. Table 2 reports the above-mentioned measurements for the seven stars. The lithium I $6708 \AA$ absorption line is visible in the spectra of 3 stars (XEST-09-042, XEST-08-003, and XEST-26-062); the same spectra show $\mathrm{H} \alpha$ emission, as well as broadened absorption lines indicative of high rotational velocities. Moreover, their radial velocity is similar to those of known Taurus members. In the light of these results detailed in the following Sects. 4.1.1, 4.1.2, and 4.1.4, we identify these stars as new pre-main sequence stars of the Taurus-Auriga region. In the spectra of the other four stars, $\mathrm{H} \alpha$ is in absorption and all absorption features are narrow.

\subsubsection{Lithium absorption}

The $E W$ of the Li I $6708 \AA$ line was measured for the stars XEST-09-042, XEST-08-003, and XEST-26-062 by numerical integration along the spectrum profile, after normalization to the continuum. For each of these measurements, errors were estimated by repeating the calculation with different levels of the continuum.

As shown in Sect. 4.1.3, XEST-09-042, XEST-08-003, and XEST-26-062 have a spectral type likely in the range K5-M3. Lithium absorption at $6708 \AA$ with $E W$ of $\sim 500-600 \mathrm{~m} \AA$ (see Table 2) implies Li abundances $A(\mathrm{Li}) \sim 2.7-3.2$ (Soderblom et al. 1990) that, in turn, place all these stars younger than $\sim 15 \mathrm{Myr}$, using the models by Siess et al. (2000).

For each of the other four stars, we give in Table 2 a rough upper limit to the $E W$ of the $\mathrm{Li}$ line. Since we do not see any absorption line at the expected wavelength of the Li line (after correction for radial velocity, see Sect. 4.1.4), we fitted with a Gaussian the weakest Fe line discernible in the spectrum, close to the expected Li line position, and set its $E W$ equal to the upper limit of the Li line. Hereafter, we will consider these four stars as non members.

\subsection{2. $\mathrm{H} \alpha$ emission}

The $\mathrm{H} \alpha$ emission line in the spectra of XEST-09-042, XEST-08-003, and XEST-26-062 (Fig. 3) was measured in the same way as for the Li line, while for the four non-members we fitted the absorption line profile with a Voigt function. We noted that the $\mathrm{H} \alpha$ line is partially saturated in all of these last stars, hence the values reported in Table 2 are likely to be overestimated.

In the spectrum of XEST-26-062, the profile of the $\mathrm{H} \alpha$ emission line is broad and asymmetric, with absorption in its central part. The full width at $10 \%$ of the peak is $\sim 320 \mathrm{~km} \mathrm{~s}^{-1}$, and surely above the limit of $\sim 270 \mathrm{~km} \mathrm{~s}^{-1}$ that divides classical from weak-lined T Tauri stars according to White \& Basri (2003) also taking into account the broadening due to the stellar rotation (see Table 2). These results clearly show that XEST-26-062 is a CTTS. The value of $E W$ for this star ( -9 $\AA$ ) may be underestimated due to the likely presence of veiling. Instead, the stars XEST-09-042 and XEST-08-003 have $E W(\mathrm{H} \alpha)$ of $-2.5 \AA$ and $-3.2 \AA$, respectively, and we have classified them as new weaklined $\mathrm{T}$ Tauri stars. 

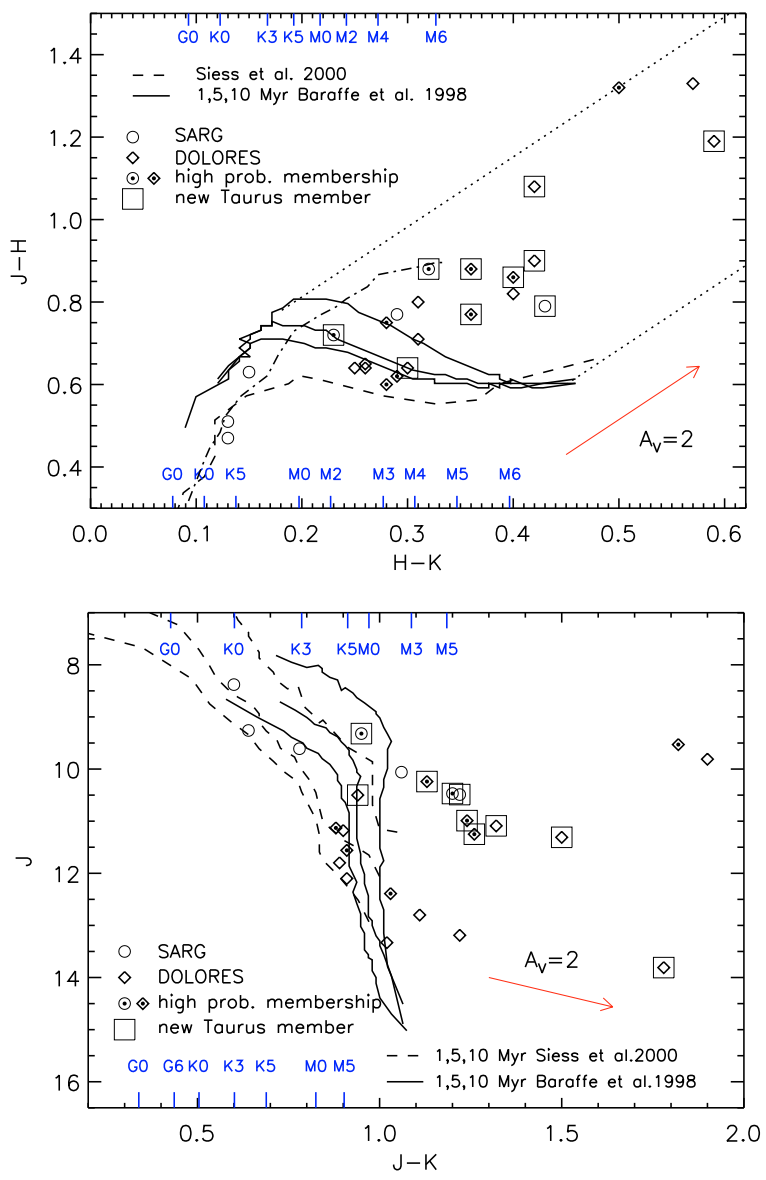

Fig. 1. Color-color and color-magnitude IR diagrams for the 25 candidates studied in this work. Circles mark stars observed with SARG, diamonds are stars observed with LRS. A small dot inside the symbol indicates a higher probability of membership (see text). New members confirmed from this work are marked with a square. Isochrones by Baraffe et al. (1998) and Siess et al. (2000), calculated for 1, 5, and $10 \mathrm{Myr}$ and at the distance of $140 \mathrm{pc}$, are shown as solid and dashed lines, respectively. In the color-color diagram, we report only the $10 \mathrm{Myr}$ isochrone by Siess et al., because all isochrones are practically coincident. In the same plot, the locus of giant stars is shown as a dot-dashed line, while the two dotted lines mark the region where stars without IR excess are expected to lie. Colors of MS stars (Kenyon \& Hartmann 1995) and giant stars (Bessell \& Brett 1988) for various spectral types are indicated, respectively, at the bottom and top of each diagram.

\subsubsection{Spectral classification}

We performed spectral classification of these 7 sources by employing the whole red region and the $\sim 200 \AA$ wide region around the 5890-5896 $\AA$ Na I doublet of our SARG spectra. We compared the sodium region and the $\sim 70 \AA$ wide spectral region centered on the $\mathrm{H} \alpha$ with high-resolution UES spectra of standard stars from Montes \& Martin (1998). Moreover, we reduced the spectral resolution of the SARG spectra to $R \sim 2400$, to compare them with low-resolution spectra included in the library by Sánchez-Blázquez et al. (2006). Following Strassmeier \& Fekel (1990), the ratio of residual intensity of Co I $6450 \AA$ and Ca I $6449 \AA$ lines allowed us to assess the luminosity class for non-members.

Spectral classification is quite secure for the K-type field stars, for which normalization to the continuum of the SARG spectra was straightforward, and their spectral lines are well-resolved. By comparison with the spectra of standard stars, errors on the spectral types were estimated to be \pm 1 sub-type. In contrast, broadened lines, uncertain continuum normalization, and veiling in the case of XEST-26-062, made the classification of the three new members more uncertain. XEST-09-042 and XEST-08-003 have very similar spectra, which suggests a mid/late-K type, while XEST-26-062 is most likely an early-M type star. Spectral types and luminosity classes of the 7 sources are reported in Table 2.

\subsubsection{Radial and rotational velocities}

Radial and rotational velocities for each of the 7 stars were measured simultaneously with the task RV within IRAF, by crosscorrelating the observed SARG spectrum with an adequate template spectrum. Template spectra at a resolution of $R=29000$ were synthesized by using ATLAS9 (Kurucz 1970) model atmospheres, with temperatures and gravities as expected for the stars analyzed here (see Sects. 4.1.3 and 5.1).

The peak of the cross-correlation function was fitted with a Gaussian plus a background level. We found that the correlation peak for the case of the new member XEST-08-003 is slightly asymmetric, which may indicate a binary nature for this star.

The relation between the FWHM of the peak and the stellar rotational velocity $v_{\text {rot }}$ was calibrated by correlating the synthesized template spectrum with artificially broadened templates for different values of stellar rotation. We noted that the $v_{\text {rot }}-F W H M$ relation is almost independent of the effective temperature and gravity of the template. Moreover, given the resolution and $S / N$ of our SARG spectra, the measurement of rotational velocities significantly below $\sim 10 \mathrm{~km} \mathrm{~s}^{-1}$ is difficult.

Table 2 contains the radial and rotational velocities for the 7 stars observed with SARG. The radial velocities of XEST-09-042, XEST-08-003, and XEST-26-062 are in very good agreement with that of the Taurus-Auriga association $\left(v_{\mathrm{rad}}=16.03 \pm 6.43 \mathrm{~km} \mathrm{~s}^{-1}\right.$, measured by Bertout \& Genova 2006, based on a sample of 127 Taurus members), and thus is another indication that these stars belong to the TMC. Their rotational velocities are typical of other young stars (see, for example, the tables in Güdel et al. 2007), while the 4 field stars are slow rotators with projected rotational velocities lower than $\sim 4-5 \mathrm{~km} \mathrm{~s}^{-1}$ and radial velocities not consistent with Taurus members.

\subsection{Low-resolution spectra}

We derived spectral types for the 18 candidate members listed in Table 1 by employing low-resolution spectra obtained with DOLORES (Fig. 4). We also inferred the presence of $\mathrm{H} \alpha$ emission in 15 of these stars, indicative of magnetic activity (and accretion in classical T Tauri stars). In Tables 3 and 4 we give a rough estimate of the $\mathrm{H} \alpha$ equivalent width, measured by integration of the spectral counts. Errors on the $E W$ are in the range $10-30 \%$, due to the limited spectral resolution and to the uncertainty on the correct continuum level.

Spectral classification was performed through visual comparison of our stellar spectra with those, at similar resolution, of standard stars of various spectral types ( $G, K$, and $M)$ and luminosity classes (dwarfs and giants) in the libraries of Jacoby et al. (1984) and Sánchez-Blázquez et al. (2006). In particular, we based our classification upon the presence and strength of the following spectral features: $\mathrm{Mg}$ I triplet at $\sim 5167-5184 \AA$ and the $\mathrm{Cr}$ I line at $\sim 5206 \AA$; the blend of Fe I, Ca I, and Ti I lines 

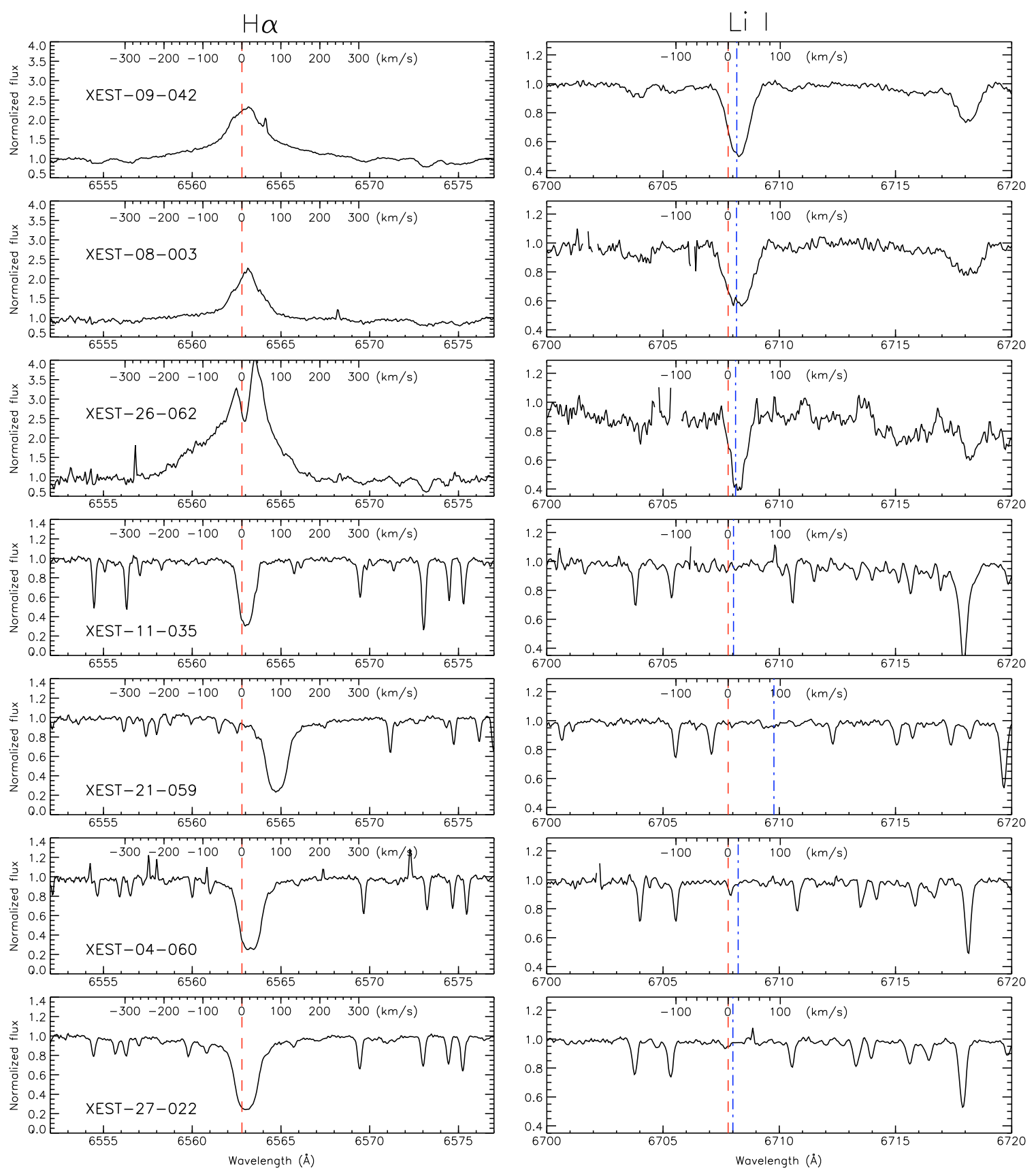

Fig. 2. High-resolution spectral regions (normalized to the continuum) centered around the $\mathrm{H} \alpha$ (left panels) and Li I $6708 \AA$ (right panels) lines. The red dashed vertical line in each plot marks the nominal wavelength of the spectral line; the blue dot-dashed lines in the right panels show the expected positions in the stellar spectrum of the Li line, based on the radial velocity shift. A velocity scale is reported on top of each panel. All prominent and narrow emission features in the plotted spectra (including that in the right side of the H $\alpha$ profile of XEST-09-042) are due to cosmic rays or hot pixels.

at 5495-5515 $\AA$; the Na I doublet at 5890-5896 ̊; the Ca I and Fe I lines in the range $\sim 6100-6200 \AA$; the large blend of Ti I, Fe, and calcium hydride $(\mathrm{CaH})$ lines in the range $\sim 6350-6410 \AA$; the unresolved Fe I, Ca I, and Ba II lines around $\sim 6495 \AA$ 象 the molecular bands $\sim 200 \AA$ wide of titanium oxide (TiO) centered around $\sim 5950 \AA$ and $\sim 6250 \AA$; and the sharp edges at $\sim 4750 \AA$, $\sim 4950 \AA, \sim 5170 \AA$, and $\sim 5450 \AA$. We also used the relative strength of the $\mathrm{H} \alpha$ and $\mathrm{H} \beta$ absorption lines in the spectra of the two G-type stars of our sample.

Low-resolution spectra of G-type stars appear relatively featureless in the spectral range $4800-6800 \AA$, with rather weak absorption lines from $\mathrm{Na}, \mathrm{Mg}$, and $\mathrm{Fe}$ and prominent $\mathrm{H} \alpha$ line as the main features; strong $\mathrm{Na}$ I doublet, $\mathrm{Mg}$ I triplet, and Ca I 6100-6200 A lines are the most distinguishing characteristics of K-type stars, while the cooler atmospheres of M-type stars are easily recognized from the $\mathrm{TiO}$ molecular bands. We identified $2 \mathrm{G}$-type stars and $2 \mathrm{~K}$-type stars, while the remaining 14 sources are classified as M-type stars. Errors on the spectral type are typically $\sim 2$ sub-types, small enough for membership purposes (see Sect. 5.2). As we show in Sect. 5.2, the G-type stars and one K-type star cannot be consistent with being pre-main sequence stars, regardless of their luminosity classes. 
Table 2. Measures of the equivalent widths of the Li I $6708 \AA$ and $\mathrm{H} \alpha$ lines and of radial and rotational velocities for the 7 stars observed with SARG.

\begin{tabular}{|c|c|c|c|c|c|}
\hline star & $\begin{array}{c}\mathrm{Li} E W \\
\AA\end{array}$ & $\begin{array}{c}\mathrm{H} \alpha E W \\
\AA\end{array}$ & $\begin{array}{c}\begin{array}{c}v_{\text {rad }} \\
\mathrm{km} \mathrm{s}^{-1}\end{array} \\
\end{array}$ & $\begin{array}{c}v_{\text {rot }} \sin i \\
\mathrm{~km} \mathrm{~s}^{-1}\end{array}$ & $\begin{array}{c}\text { Spectral } \\
\text { classification }\end{array}$ \\
\hline \multicolumn{6}{|c|}{ new TMC members } \\
\hline XEST-09-042 & $0.53 \pm 0.03$ & $-3.2 \pm 0.5$ & $16.7 \pm 1.0$ & $31.3^{+2.8}$ & K5-7 V/IV \\
\hline XEST-08-003 & $0.56 \pm 0.05$ & $-2.5_{-0.7}^{+0.4}$ & $16.2 \pm 1.5$ & $44_{-3}^{+4}$ & K5-7 V/IV \\
\hline XEST-26-062 ${ }^{a}$ & $0.46 \pm 0.06$ & $-9.0 \pm 1.5$ & $14.4 \pm 1.1$ & $18 \pm 3$ & M \\
\hline \multicolumn{6}{|c|}{ non members } \\
\hline XEST-11-035 & $<0.03$ & $0.76_{-0.06}^{+0.15}$ & $10.39 \pm 0.25$ & $<4$ & K5 V \\
\hline XEST-21-059 & $<0.025$ & $1.5 \pm 0.2$ & $88.10 \pm 0.23$ & $<5$ & K1 IV/III \\
\hline XEST-04-060 & $<0.025$ & $1.4 \pm 0.2$ & $19.34 \pm 0.23$ & $<5$ & K1 IV/III \\
\hline XEST-27-022 & $<0.02$ & $1.45 \pm 0.15$ & $9.13 \pm 0.19$ & $<5$ & K1 IV/III \\
\hline
\end{tabular}

${ }^{a}$ Absolute values of the equivalent widths of the $\mathrm{Li}$ and $\mathrm{H} \alpha$ lines are likely underestimated since veiling was not accounted for.
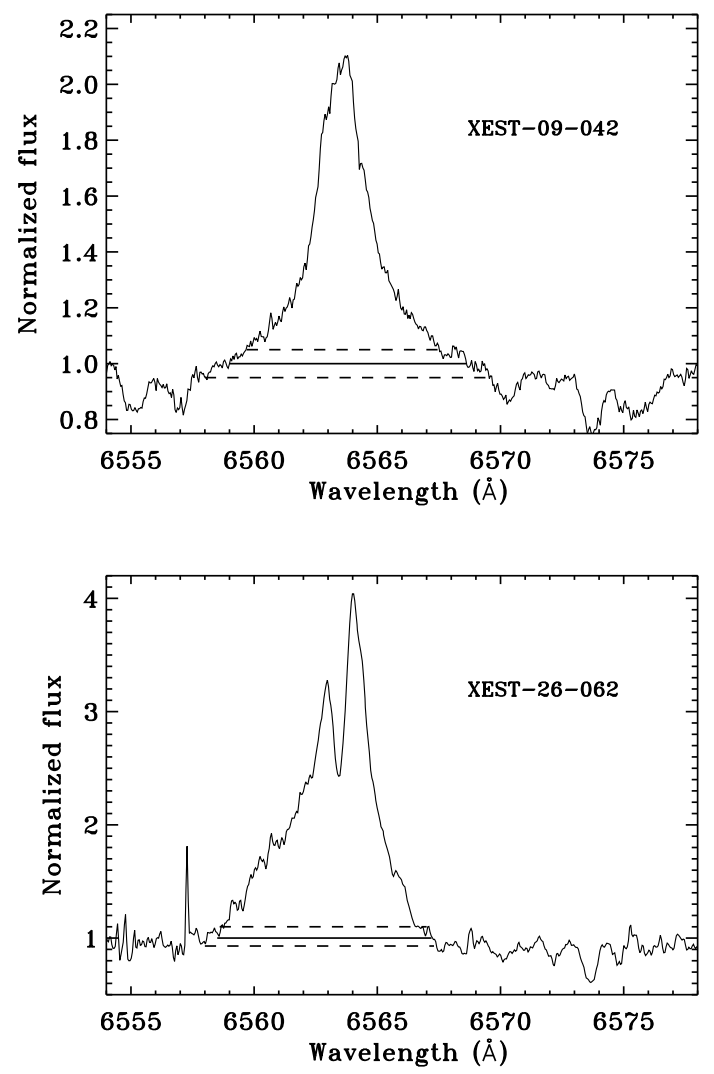

Fig. 3. Measurement of the $\mathrm{H} \alpha$ line in the spectra of XEST-09-042 and XEST-26-062 normalized to the continuum. The three horizontal lines represent the three different levels of continuum emission assumed for the measurement of the $E W$ (solid line) and the estimate of the errors (dashed lines).

Instead, the K-type star XEST-11-078 shows $\mathrm{H} \alpha$ emission with $E W \sim-16.5 \AA$, atypical in active K-type dwarfs. In its spectrum we also identified the [O I] $6300 \AA$ and [N II] $6583 \AA$ emission lines, typical of young accreting stars, so hereafter we consider XEST-11-078 a new classical T Tauri star belonging to Taurus-Auriga.

For the M-type sources, it is crucial to assess their luminosity class, in order to distinguish between MS or giant field stars and pre-main sequence objects. Two well-marked spectral features in our DOLORES spectra can be reliably used for gravity diagnostics, namely the $\mathrm{CaH}$ band ( 6380-6390 А), to be compared with the close absorption feature at $\sim 6360 \AA$ due to Ti and Fe lines, and the sodium doublet (5890-5896 ̊). In Fig. 5 we zoom in on such spectral regions for our M-type stars, and show for comparison the same regions in the spectra of standard dwarfs and giants from the library of Sánchez-Blázquez et al. (2006), smoothed at the same resolution. Sodium absorption is strong in standard dwarfs of all sub-types, while it weakens considerably in giants from M2 towards later spectral types. The $\mathrm{CaH}$ band is stronger than the adjacent $\mathrm{Ti}$ and $\mathrm{Fe}$ absorption feature in dwarfs later than M0; on the contrary, it is much weaker than the Ti and Fe blend in giants of all sub-types.

First, we observe that 5 stars labeled in Fig. 5 as "field stars" (XEST-22-111，XEST-12-012，XEST-27-084，XEST-06-041, and XEST-15-075) show prominent absorption from sodium and $\mathrm{CaH}$, which closely resemble the spectra of standard dwarfs. As stated in Sect. 5.2, these stars are compatible with being MS field stars.

The 6 stars labeled as "new Taurus members" (XEST-08033, XEST-08-047, XEST-08-049, XEST-11-087, XEST-16-045 and XEST-17-059) differ markedly from the stars of the previous group and the standard dwarfs. In fact, sodium absorption is much weaker and the $\mathrm{CaH}$ band, compared with the $6360 \AA \mathrm{ab}-$ sorption feature, shows intermediate behavior between dwarfs and giants, as one could expect for intermediate-gravity stars like pre-main sequence objects. We stress that both $\mathrm{Na}$ I and $\mathrm{CaH}$ lines consistently indicate a gravity lower than in MS stars for these stars. Such information, together with their consistent position with pre-main sequence models in IR color-color and color-magnitude diagrams (Sect. 5.2), lead to the conclusion they are young objects belonging to the TMC.

XEST-15-034 shows signs of high gravity, while the situation is less clear for XEST-08-014 and XEST-20-071 from the behavior of the $\mathrm{Na}$ I lines and $\mathrm{CaH}$ band. These three stars are labeled as "uncertain" in the figure, for reasons also discussed in Sect. 5.2.

\section{IR diagrams, membership, and stellar properties}

The IR color-color and color-magnitude diagrams were employed to derive information about the stellar properties and the interstellar absorption $A_{\mathrm{V}}$ and to assess membership in the case of the stars observed at low resolution. Using the $J, H$, and $K$ magnitudes from the 2MASS catalog, we plotted the sources in the $J$ vs. $J-K, H$ vs. $H-K$, and $J-H$ vs. $H-K$ diagrams, together with isochrones and the main sequence calculated for the distance of the TMC (140 pc). 


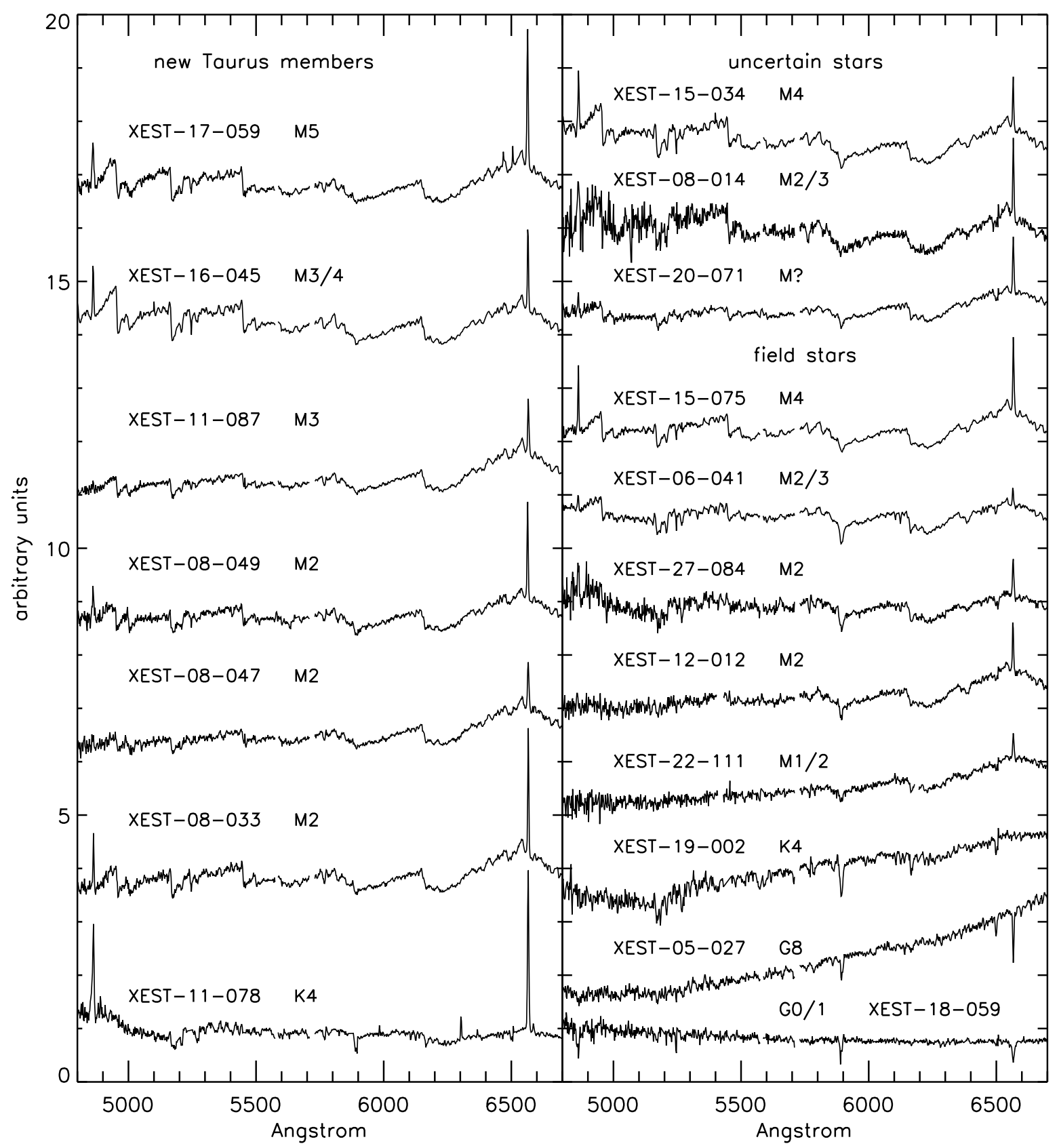

Fig. 4. Low-resolution spectra of the 7 new Taurus members (left), the three uncertain cases (upper right) and the field stars (lower right). Recall that these spectra are not corrected for the instrumental response and air mass effects.

We assumed that the stars analyzed here have no significant IR excess from a circumstellar disk, and therefore estimates of $A_{\mathrm{V}}$ can be derived simply by shifting back their positions in the color-color diagram to PMS isochrones, or to the MS or the giant locus along the reddening vector (Fig. 6). We discuss individual cases in Sects. 5.1 and 5.2 where we know, or we suspect, this assumption is not true.

The results reported in this section were obtained using isochrones and evolutionary tracks by Baraffe et al. (1998), but we also verified that our overall conclusions do not change substantially if we use the models by Siess et al. (2000).

Distances of main sequence field stars were calculated by matching the dereddened positions with the MS, scaled for the distance, both in the $J$ vs. $J-K$ and in the $H$ vs. $H-K$ diagrams, and averaging the two values, if different.
Masses and X-ray luminosities were also estimated. The method used to derive masses and relevant errors for PMS stars is described in Sects. 5.1 and 5.2. Masses of MS stars have been evaluated from dereddened IR colors and the MS by Baraffe et al. (1998). For X-ray luminosities, we used the relation between $L_{\mathrm{X}}, N_{\mathrm{H}}$ and the EPIC/PN count-rate found for known TMC members (Scelsi et al. 2007). We have verified that such a relation approximately holds also for coronae cooler $(T \sim$ $0.3 \mathrm{keV}$ ) than those of young active stars, if $N_{\mathrm{H}} \lesssim 10^{22} \mathrm{~cm}^{-2}$, so it was adopted for computing X-ray luminosities of both new TMC members and MS field stars. For giants (or subgiants) field stars, we evaluated only the $L_{\mathrm{X}} / L_{\text {bol }}$ ratios (using the tables of intrinsic colors from Zombeck 1991).

Rough estimates of the errors on extinctions, masses, and distances of MS field stars were calculated by propagating the errors on the IR colors and the spectral types. 
Table 3. Stellar properties for the new Taurus members and the uncertain sources observed with DOLORES.

\begin{tabular}{lccccccc}
\hline \hline Star & Sp. type & $\begin{array}{c}\mathrm{H} \alpha E W^{a} \\
(\AA)\end{array}$ & $\begin{array}{c}A_{\mathrm{V}}{ }^{b} \\
(\mathrm{mag})\end{array}$ & $\begin{array}{c}N_{\mathrm{H}}{ }^{c} \\
\left(10^{21} \mathrm{~cm}^{-2}\right)\end{array}$ & $\begin{array}{c}M \\
\left(M_{\odot}\right)\end{array}$ & $\begin{array}{c}\text { Age (range) } \\
(\mathrm{Myr})\end{array}$ & Evol. stage \\
\hline XEST-08-033 & M2 IV & -11 & $1.8 \pm 0.1$ & $4.0 \pm 1.0$ & $0.35_{-0.15}^{+0.1}$ & $5(1-10)$ & WTTS \\
XEST-08-047 & M2 IV & -6 & $3.2 \pm 0.3$ & & $0.50_{-0.05}^{+0.1}$ & $7(5-10)$ & WTTS \\
XEST-08-049 & M2 IV & -9 & $1.8 \pm 0.2$ & $3.0 \pm 0.2$ & $0.50_{-0.15}^{+0.05}$ & $5(3-10)$ & WTTS \\
XEST-11-078 & K2-4 V & -16.5 & - & & - & - & CTTS \\
XEST-11-087 & M3 IV & -6 & $2.0 \pm 0.2$ & & $0.25 \pm 0.1$ & $3(1-5)$ & WTTS \\
XEST-16-045 & M3/4 IV & -8.5 & $0(<0.1)$ & \multicolumn{7}{c}{$0.30 \pm 0.1$} & $3(1-5)$ & WTTS \\
XEST-17-059 & M5 IV & -16 & $0.6 \pm 0.1$ & $1.8 \pm 0.3$ & $0.20 \pm 0.05$ & $1(<3)$ & WTTS \\
\hline & \multicolumn{7}{c}{ uncertain stars } \\
XEST-08-014 & M2/3 & -8.5 & $\sim 0$ & $\lessgtr 0.2$ & 0.4 & $\sim 10$ & WTTS or MS at $\sim 65$ pc \\
XEST-15-034 & M4 V & -5 & $\sim 0-0.15$ & $\lessgtr 0.2$ & 0.35 & $\gtrsim 10$ & WTTS or MS at $\sim 120$ pc \\
XEST-20-071 & M? & -6 & - & $4.1 \pm 0.4$ & - & - & $?$ \\
\hline
\end{tabular}

${ }^{a}$ From low-resolution spectra (Sect. 4.2); ${ }^{b}$ from IR color-color diagram (Sect. 5.2); ${ }^{c}$ from X-ray spectral fitting (Scelsi et al. 2007); ${ }^{d}$ possible IR excess may affect the $A_{\mathrm{V}}$, mass and age values; ${ }^{e}$ values of $A_{\mathrm{V}}, M$ and age refer to the PMS case and are not confirmed.

Table 4. Stellar properties for the confirmed field stars observed with DOLORES.

\begin{tabular}{lccccccc}
\hline \hline Star & Sp. type $^{a}$ & $\begin{array}{c}\mathrm{H} \alpha E W^{a} \\
(\AA)\end{array}$ & $\begin{array}{c}A_{\mathrm{V}}{ }^{b} \\
(\mathrm{mag})\end{array}$ & $\begin{array}{c}N_{\mathrm{H}}{ }^{c} \\
\left(10^{21} \mathrm{~cm}^{-2}\right)\end{array}$ & $\begin{array}{c}M \\
\left(M_{\odot}\right)\end{array}$ & $\begin{array}{c}d \\
(\mathrm{pc})\end{array}$ & Evol. stage \\
\hline XEST-05-027 & G5 IV/III & 2 & $7.5 \pm 0.3$ & $11.0 \pm 1.5$ & - & - & giant \\
XEST-06-041 & M2/3 V & -1.5 & $0.45_{-0.3}^{+0.2}$ & & $0.50 \pm 0.05$ & $120 \pm 20$ & MS \\
XEST-12-012 & M2 V & -5 & $2.2 \pm 0.3$ & & $0.50 \pm 0.05$ & $155_{-15}^{+30}$ & MS \\
XEST-15-075 & M4 V & -7 & $0.3 \pm 0.3$ & & $0.50 \pm 0.05$ & $115 \pm 15$ & MS \\
XEST-18-059 & G0/1 & 2.8 & $3.1_{-0.7}^{+0.4}$ & & $1.15 \pm 0.15$ & $330_{-60}^{+160}$ & MS (or giant?) \\
XEST-19-002 & K4 V & 0 & $3.3 \pm 0.7$ & & $0.8 \pm 0.1$ & $400_{-100}^{+100}$ & MS \\
XEST-22-111 & M1/2 V & -3.5 & $1.7 \pm 0.4$ & & $0.55 \pm 0.05$ & $175 \pm 30$ & MS \\
XEST-27-084 & M2 V & -4.5 & $1.2 \pm 0.3$ & $0.7 \pm 0.4$ & $0.55 \pm 0.05$ & $150 \pm 25$ & MS \\
\hline
\end{tabular}

${ }^{a}$ From low-resolution spectra (Sect. 4.2); ${ }^{b}$ from IR color-color diagram (Sect. 5.2); ${ }^{c}$ from X-ray spectral fitting (Scelsi et al. 2007); ${ }^{d}$ assuming main sequence star.

\subsection{Stars observed with SARG}

The results shown in Sect. 4.1 confirm membership to the TMC of XEST-09-042, XEST-08-003, and XEST-26-062, for which we derived here estimates of visual absorption, mass, and age. The four remaining sources are one foreground main sequence stars and three K1 IV/III stars behind the cloud.

We shifted the new PMS stars in the color-color diagram along the reddening vector back to the isochrones at 1, 5, and 10 Myr (see upper panel of Fig. 6 for the example case of XEST-08-003), and then corrected their positions in the color-magnitude diagrams. We visually assigned the mass, age, and $A_{\mathrm{V}}$ values, and relevant ranges, that give the best agreement between the three diagrams (middle and lower panels of Fig. 6).

The weak-lined XEST-09-042 shows relatively low absorption $(\leq 0.3 \mathrm{mag})$, roughly consistent with the $N_{\mathrm{H}} \sim 10^{21} \mathrm{~cm}^{-2}$ measured from spectral fitting of the X-ray spectrum. We estimated the mass of this $\operatorname{star} M=0.6 \pm 0.1 M_{\odot}$ and its age to be $\sim 3 \mathrm{Myr}$, in the range 1-5 Myr.

For the weak-lined XEST-08-003, rather good agreement between the three diagrams is found assuming a spectral type early-M and $A_{\mathrm{V}}=1.5 \pm 0.2$, consistent with the X-ray measure of $N_{\mathrm{H}}\left(2.4 \times 10^{21} \mathrm{~cm}^{-2}\right)$. The mass is estimated to be $0.45 M_{\odot}$ (in the range $0.35-0.65 M_{\odot}$ ) and the age $\sim 5 \mathrm{Myr}$ (in the range 3-7 Myr). We recall that this source is likely a binary star (Sect. 4.1.4), hence the IR photometry may include contribution from more than a star. Therefore, the mass and age of this TMC member remain more uncertain.

The same procedure described above was applied to the new CTTS member XEST-26-062, giving mass $0.25 \pm 0.05 M_{\odot}$, age $\sim 1(\lesssim 3) \mathrm{Myr}$, and $A_{\mathrm{V}}=1.0 \pm 0.1$. However, IR excess from the disk is not accounted for, so the mass of this M-type star may be slightly greater than the value reported here.

The star XEST-11-035 shows little absorption $\left(A_{\mathrm{V}} \sim 0.1\right)$ from the color-color diagram, and is most likely an old $\sim$ K5 main sequence $\operatorname{star}\left(M \sim 0.7 M_{\odot}\right)$ located at a distance of $\sim 80 \mathrm{pc}$ from us, with low $L_{\mathrm{X}}\left(\sim 3 \times 10^{27} \mathrm{erg} \mathrm{s}^{-1}\right)$. The K1 IV/III stars XEST-27-022, XEST-21-059, and XEST-04-060 show visual absorption of $\sim 0.5,0.5$, and 2.8 , respectively; they have $L_{\mathrm{X}} / L_{\mathrm{bol}} \sim 0.5-1 \times 10^{-5}$.

\subsection{Stars observed with DOLORES}

For the stars observed at low resolution we used the IR diagrams with the aim of providing further support of their nature as young objects or field stars, and deriving useful stellar properties as in Sect. 5.1. We do not consider the new CTTS XEST-11-078 here, since its position in IR diagrams is probably affected by significant IR excess. Despite the uncertainties of $\sim 2$ subtypes in the spectral type derived from DOLORES spectra, we can distinguish M-type stars from G- and K-type stars. This is sufficient for membership confirmation because we can univocally identify the intersection between the dereddening vector of an 


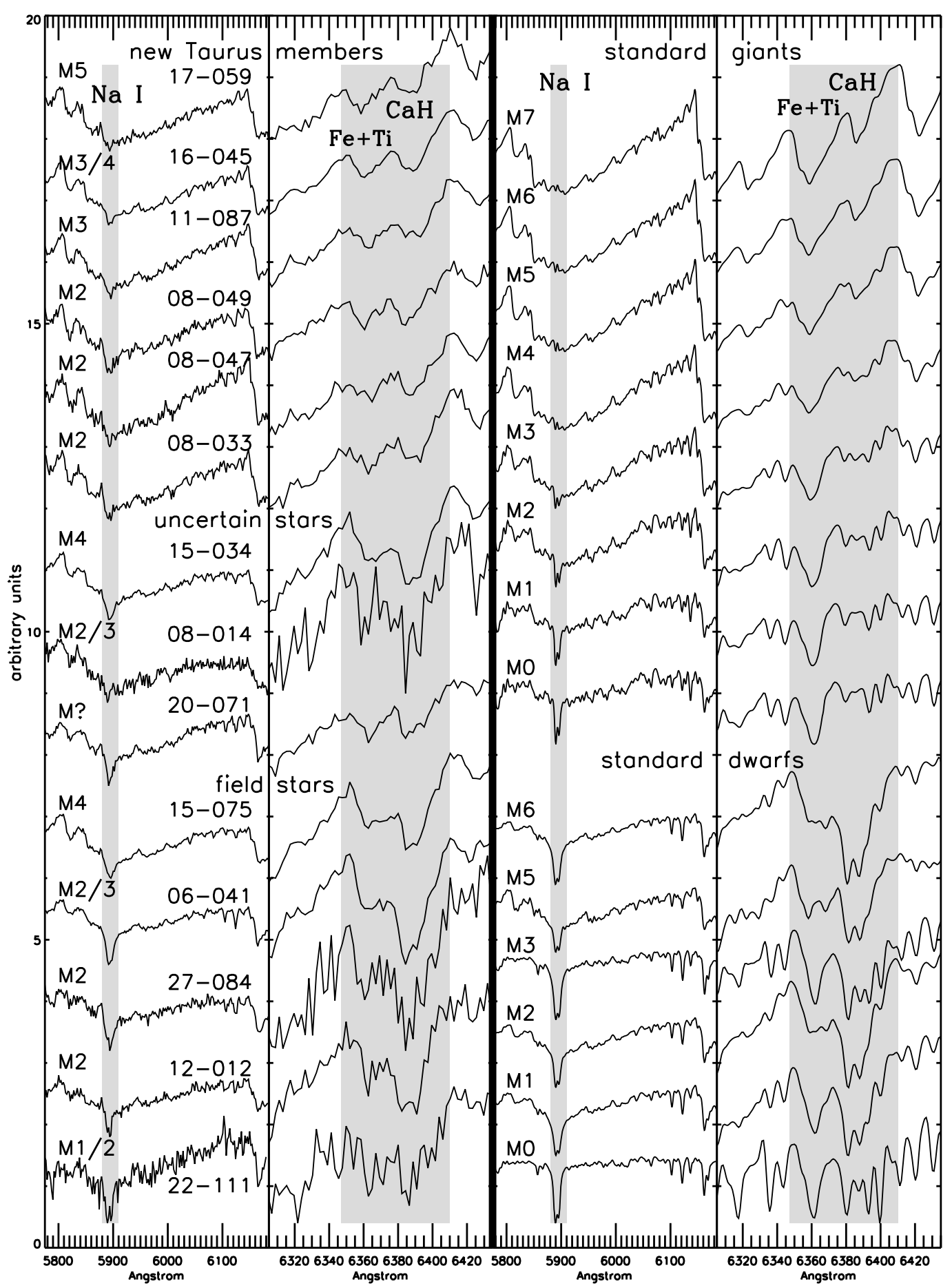

Fig. 5. Left panels: zooms of DOLORES spectra of the 7 new Taurus members (up), the three uncertain cases (middle) and the field stars (bottom), showing the Na I doublet $(\sim 5890 \AA)$ and the $\mathrm{Fe}+\mathrm{Ti}(\sim 6360 \AA)$ and $\mathrm{CaH}(\sim 6390 \AA)$ absorption features. Right panels: same spectral regions for a sequence of M-type standard giants (up) and dwarfs (bottom).

absorbed star with a given evolutionary model. We divide the whole LRS sample into 3 different groups (6 new TMC members, 3 uncertain cases, and 8 field stars), as explained in the following.

\subsubsection{New TMC members}

The first group is composed of the 6 M-type stars XEST-08-033, XEST-08-047, XEST-08-049, XEST-11-087, XEST-16-045, and
XEST-17-059 whose low-resolution spectra (Sect. 4.2) indicate gravity lower than MS stars of the same spectral type. In any case, we first explored the hypothesis that these are indeed field stars. We can exclude these M-type stars being giants behind the cloud, because their position in the color-color diagram is inconsistent with the locus of giants, with the exception of XEST-08-047, and because X-ray emitting M-type giants are very unusual (see, e.g. Maggio et al. 1990; Hunsch et al. 1998). We can also exclude these stars being active MS stars, because 

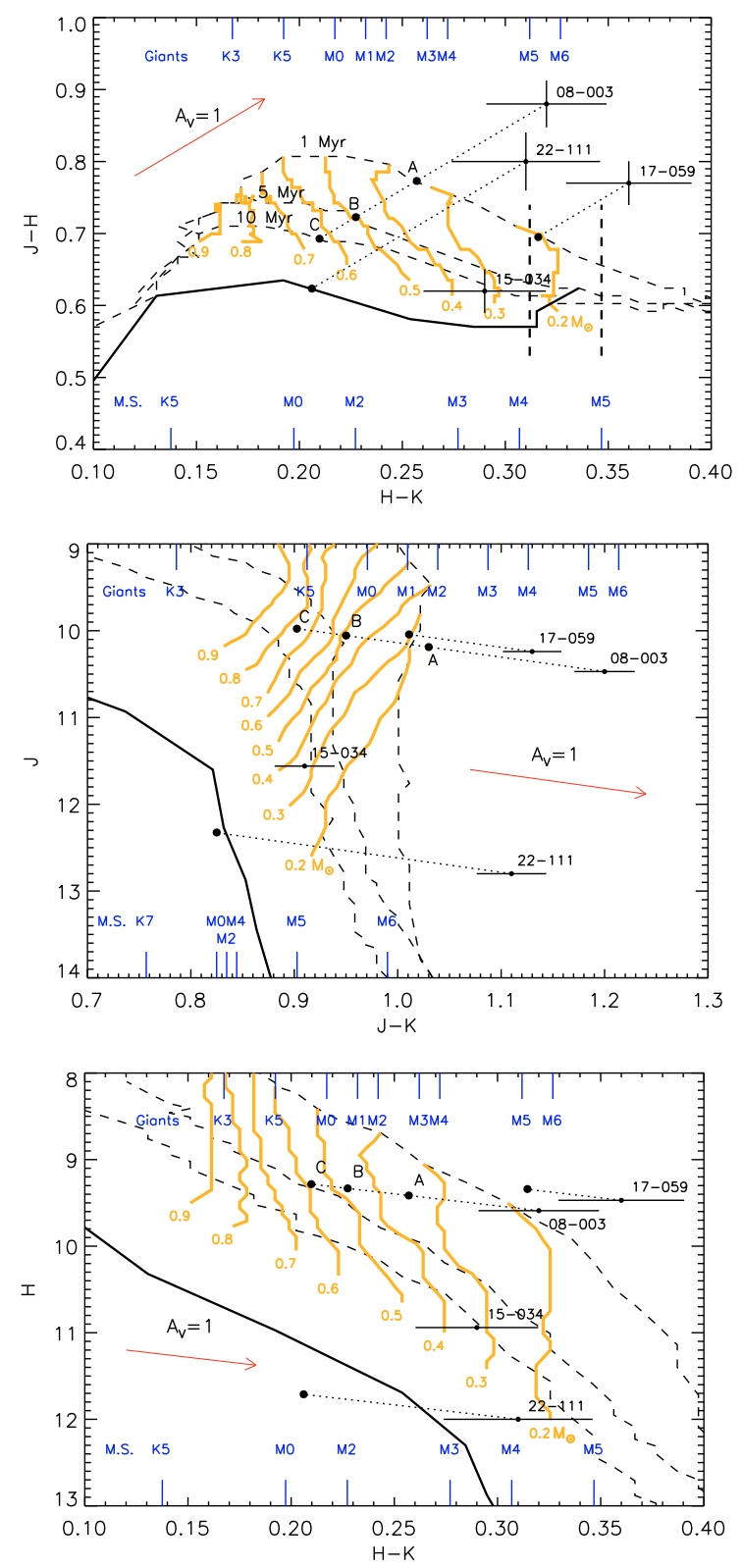

Fig. 6. Color-color (upper panel) and color-magnitude diagrams (middle and lower panels) illustrating the method used to determine stellar properties and membership. In each plot, the thin dashed lines are isochrones at 1, 5, and $10 \mathrm{Myr}$; the thick solid line is the MS. Light (yellow in the online version) solid lines are evolutionary tracks for different masses. The two vertical dashed lines in the color-color diagram mark the $H-K$ range expected for an M5 PMS star as XEST-17-059 (see Sect. 5.2). The points A-C mark the dereddened positions of the new TMC member XEST-08-003 on the isochrones in the color-color diagram, and the positions in the color-magnitude diagrams for relevant $A_{\mathrm{V}}$ values. The field star XEST-22-111 is shifted back to the MS in the color-color diagram. XEST-15-034 is an uncertain case.

in this hypothesis dereddening onto the MS track would give significantly greater visual absorption than allowed by the small derived photometric parallaxes.

With the method described below, we found that these six stars have dereddened positions in the IR diagrams that are compatible with PMS stars. For a given star belonging to this group, we marked in the color-color diagram the $H-K$ color range whose extremes are defined by the $H-K$ colors of MS (from
Kenyon \& Hartmann 1995) and giant (from Bessell \& Brett 1988) stars of the same spectral type of that star, as classified in Sect. 4.2. Then, we dereddened the star position in this diagram searching for "PMS solutions" with intrinsic $H-K$ color within the above-mentioned range (Fig. 6). Note that intrinsic colors of PMS stars depend on the stellar age, which we do not know at this stage. Also, the $J-H$ color may be a better choice than the $H-K$ color to avoid overestimating the extinction in the case of optically thick disks. However, we preferred to use the $H-K$ color because $J-H$ shows variations with the age, for a given spectral type, greater than the former color, thus providing poor constraints; moreover, the consistency of the results from IR diagrams and spectral types form LRS spectroscopy (as well as $\mathrm{H} \alpha$ emission level) indicates that these stars have no significant IR excess, with the possible exception of XEST-11-087 (see below). With this method, we eventually found the best agreement between the three diagrams for the $A_{\mathrm{V}}$, mass, and age values reported in Table 3. We estimated errors on these quantities by taking into account the errors on the IR colors and the LRS-derived spectral type. We note that the spectral type obtained from the color-color diagram for XEST-11-087 is somewhat later than what is derived from the LRS spectrum, which may indicate the presence of significant IR contribution from a disk.

Therefore, on the basis of all these results and the $\mathrm{H} \alpha$ equivalent widths (Table 3), we consider these stars as new members of the TMC, and we classify them as weak-lined T Tauri stars.

\subsubsection{Uncertain cases}

The second group includes the three "uncertain" stars XEST-08-014, XEST-15-034, and XEST-20-071 that deserve further investigation. The first two are consistent with being either $\sim 10$ Myr old Taurus members or main sequence stars in front of the cloud, if we conservatively consider both the errors on the LRS-derived spectral type and the IR data (Fig. 6) and that the gravity of old ( $\sim 10 \mathrm{Myr}) \mathrm{T}$ Tauri stars is much more similar to that of MS stars than giants (see the case of XEST-15034 in Fig. 5). Instead, the nature of the M-type star XEST-20071 remains uncertain. Its spectrum shows signs of having gravity higher than in a giant star. However, consistency between the three IR diagrams could be found neither as an MS nor a PMS star. From the color-color diagram, a K spectral type is indicated, which is inconsistent with the result from the lowresolution spectrum.

\subsubsection{Field stars}

Finally, 8 sources are identified as field stars. XEST-06-041, XEST-12-012, XEST-15-075, XEST-19-002, XEST-22-111, and XEST-27-084 have gravity typical of main sequence stars, and we found consistency between the three diagrams assuming they are indeed MS stars ${ }^{2}$ (Fig. 6). Although we were not able to assess the luminosity class of the early-G star XEST-18-059, its dereddened position surely falls below the main sequence, so it is excluded as a PMS object. XEST-18-059 may be a G0/1 MS star $\sim 330$ pc away, with a mass slightly greater than the solar value and $L_{\mathrm{X}} \sim 6 \times 10^{29} \mathrm{erg} \mathrm{s}^{-1}\left(L_{\mathrm{X}} / L_{\mathrm{bol}} \sim 6 \times 10^{-5}\right)$. The luminosity class of the late-G star XEST-05-027 is also not clear from its LRS spectrum, however it is excluded as an MS star because

2 Dereddening in the color-color diagram onto the $10 \mathrm{Myr}$ isochrone, under the assumption of high-gravity T Tauri star, leads to positions in the color-magnitude diagrams significantly below this isochrone. 
of the incompatibility between the distance it would have in this case $(\sim 50-60 \mathrm{pc})$ and the high visual absorption $A_{\mathrm{V}} \sim 7.5 \mathrm{mag}$ (consistent with the X-ray measure of $N_{\mathrm{H}}=1.1 \times 10^{22} \mathrm{~cm}^{-2}$ ). If XEST-05-027 were a PMS star, it would be much younger than 1 Myr from the IR diagrams, and probably would have significant IR excess. This source is included in the catalog of the Taurus Spitzer Legacy Project (Data Release 1, Padgett et al. 2006), and the SED we derived from Spitzer and 2MASS photometry shows no signs of IR excess from circumstellar material, but it is instead typical of normal stars. Therefore, XEST-05-027 is most likely a giant (or subgiant) behind the TMC, having $L_{\mathrm{X}} / L_{\text {bol }} \sim 2 \times 10^{-4}$.

Table 3 summarizes the stellar properties derived in this work for new TMC members and uncertain sources, while Table 4 gives information about the field stars.

\section{Discussion}

In this work we employed optical spectroscopy, IR photometry, and X-ray data to characterize a sample of 25 sources, previously identified as candidate pre-main sequence stars. We confirm membership in the Taurus-Auriga star-forming region for 10 of them, while 12 sources are identified as main sequence or older stars in front of or behind the cloud. Three sources remain as uncertain cases. It is worth noting that we have confirmed membership for 5 candidates out of 9 with a high probability assigned by Scelsi et al. (2007) on the basis of the X-ray analysis; 3 more are the uncertain cases, and only one such source (XEST-27-084) has been identified as an active M-type MS star. The percentage of new Taurus members with respect to the number of observed stars is at least $40 \%$, up to $\sim 50 \%$ depending on the nature of the uncertain sources. Similar percentages of confirmed new TMC members have been obtained in other works previously mentioned (Sect. 1), where candidate selection was based on optical and infrared photometry. Hence, our work indicates that candidate selections based on X-ray emission and infrared photometry are as effective as selections based on optical and infrared photometry. We also note that higher percentages of confirmed members could be obtained if the selection is made considering only X-ray sources within the $M-L_{\mathrm{X}}$ relation discussed below.

It is also interesting that 5 out of 10 newly discovered TMC members (i.e. the three lithium stars and 2 members observed at low-resolution) are variable in X-rays (see Table 1). Short-term X-ray variability, often associated to flares, is a frequent characteristic of active stars (Güdel et al. 2003; Wolk et al. 2005; Caramazza et al. 2007). The X-ray variability of pre-main sequence stars in the Taurus molecular cloud has recently been studied by Stelzer et al. (2007), who found that about half of a sample of 122 members detected in the XEST survey are variable. Our result is therefore consistent with the percentage of $\mathrm{X}$-ray variable members in Taurus.

As explained in Sects. 4.1 and 5.2.1, we found 8 new WTTS and 2 new CTTS, which is not surprising considering the initial X-ray-based candidate selection. The T Tauri type has been deduced from the strength of the $\mathrm{H} \alpha$ emission and the shape of the line profile for the stars observed with SARG. The stars XEST-08-003, XEST-08-033, XEST-08-047, XEST-16-045, and XEST-17-059 also appear in the catalog of the Taurus Spitzer Legacy Project (Data Release 1); the photometry obtained with the IRAC and MIPS cameras onboard the Spitzer Space Telescope, together with the 2MASS measurements, gives SEDs typical of stars without significant IR excess for circumstellar material, and thus confirms their nature as weak-lined T Tauri stars.
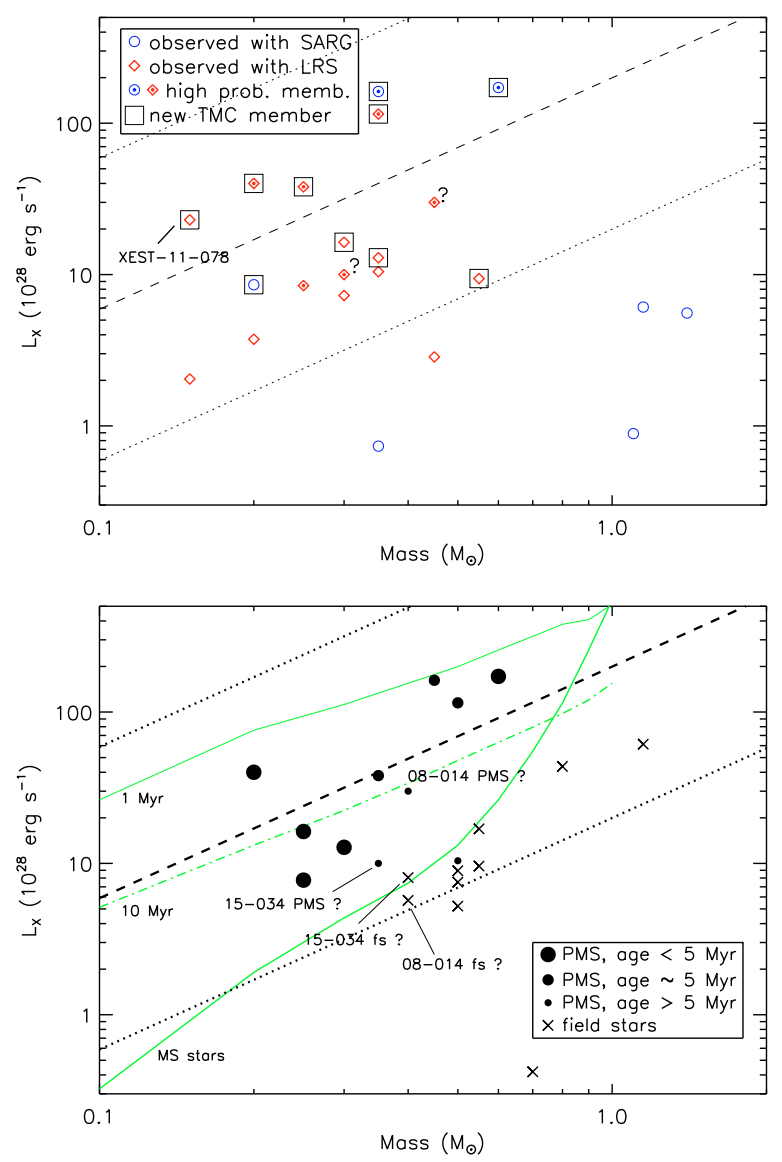

Fig. 7. Upper panel: positions of the candidates in the $M-L_{X}$ diagram, with masses and X-ray luminosities estimated before the TNG observations, assuming they are all members. Circles and diamonds mark stars observed with SARG and DOLORES, respectively. A dot inside the symbol means the candidate had a higher probability of membership on the basis of X-ray data. Squares indicate new members confirmed from this work, and "?" mark uncertain cases. The dashed line is the best fit to the data for known TMC members and the dotted lines bracket the region where known members are found. Lower panel: $M-L_{\mathrm{X}}$ diagram updated after the TNG observations. Circles mark new members from this work, with circle size indicating age, and crosses main sequence field stars. For the uncertain cases XEST-15-034 and XEST-08-014 both positions as a PMS or a field star are plotted. Light green lines are the saturation at $1 \mathrm{Myr}, 10 \mathrm{Myr}$, and in the main sequence.

The new members have spectral types in the range late-K-M so are low-mass stars, with masses estimated in the range $\sim 0.2-0.6 M_{\odot}$. These members fall in the same bins where the mass distribution of the $\sim 150$ known Taurus members (within the same XMM fields) shows its bulk. However, the 25 sources observed at the TNG and analyzed here are the optically brightest stars of the initial sample selected by Scelsi et al. (2007), and therefore other new members with masses of $\sim 0.1 M_{\odot}$, may be hidden among the 32 remaining candidates. Indeed, about 20 of them are very-low mass candidates $\left(M \lessgtr 0.2 M_{\odot}\right)$, and about ten of them are brown dwarf candidates ${ }^{3}$, which will be studied in the continuation of our optical follow-up program.

Figure 7 shows the $M-L_{\mathrm{X}}$ diagram for the sources studied in this work, for which mass estimates have been possible. In the upper panel, masses and X-ray luminosities were estimated by Scelsi et al. (2007) before the TNG observations

\footnotetext{
3 The XEST has detected 8 out of 16 known brown dwarfs included in the survey.
} 
and under the assumption that the candidates were indeed TMC members ${ }^{4}$. In this plot we report the relation $\log L_{\mathrm{X}}=$ $1.54 \log M+30.31$, best-fitting the data for known TMC members within the fields of the XEST survey (Güdel et al. 2007), and we also mark the new Taurus members from this study. All new TMC members are found within the relation derived from known members, while the five sources outside this relation (i.e. XEST-18-059, XEST-27-022, XEST-21-059, XEST-04-060, and XEST-11-035) are confirmed as non-members. This result both confirms the validity of a correlation between mass and X-ray luminosity for the young stars of Taurus and suggests that the $M-L_{\mathrm{X}}$ diagram may be used in future searches for new premain sequence stars as a further criterion for the identification of new candidate members.

The lower panel of the same figure updates the previous plot after the observations at the TNG and the analysis presented in this paper. In this plot, the new members are also distinguished by their age, as calculated in Sects. 5.1 and 5.2. We did not include giant or subgiant field stars, since their masses and $\mathrm{X}$-ray luminosities were not estimated. We marked the saturation curves $L_{X}=10^{-3} L_{\text {bol }}$ relevant to different evolutionary stages, i.e. stars at $1 \mathrm{Myr}$, at $10 \mathrm{Myr}$, and on the main sequence (the relation between bolometric luminosity and mass was taken from the models by Baraffe et al. 1998). Interestingly, the slopes of the theoretical saturation curves for PMS stars are very similar to the slope of the $M-L_{\mathrm{X}}$ relation found for Taurus members, suggesting that part of the observed spread around this best-fit relation is due to saturated stars at different ages. However, both the data in the plot and the narrower $L_{\mathrm{X}}$ range spanned by the saturation curves in the 1-10 Myr range suggest that other factors may play a role, such as flares, non-saturated stars (slow rotators), and the possibly lower $L_{X}$ of CTTS with respect to WTTS (Preibisch et al. 2005; Briggs et al. 2007).

Finally, it is also interesting to note that the saturation curve for main sequence stars steepens at $M \sim 0.5-0.6 M_{\odot}$, and explains why older stars may be found within the relation for TMC members. In fact, most of our confirmed field stars are close to, or in, the saturation regime.

Acknowledgements. We thank the referee, Dr. J. Alcalá, for his comments that helped to improve the quality of the paper. We acknowledge financial contribution from contract ASI-INAF I/088/06/0. L.S. thanks J. Lopez-Santiago and F. Damiani for useful discussions, and the contact astronomer at the TNG who performed the observations in service mode. This publication makes use of data products from the Two Micron All Sky Survey (2MASS), which is a joint project of the University of Massachusetts and the Infrared Processing and Analysis
Center/California Institute of Technology, funded by the National Aeronautics and Space Administration and the National Science Foundation.

\section{References}

Baraffe, I., Chabrier, G., Allard, F., \& Hauschildt, P. H. 1998, A\&A, 337, 403 Bertout, C., \& Genova, F. 2006, A\&A, 460, 499

Bessell, M. S., \& Brett, J. M. 1988, PASP, 100, 1134

Briceño, C., Luhman, K. L., Hartmann, L., Stauffer, J. R., \& Kirkpatrick, J. D. 2002, ApJ, 580, 317

Briggs, K. R., Güdel, M., Telleschi, A., et al. 2007, A\&A, 468, 413

Caramazza, M., Flaccomio, E., Micela, G., et al. 2007, A\&A, 471, 645

Feigelson, E. D., \& Montmerle, T. 1999, ARA\&A, 37, 363

Goodwin, S. P., Whitworth, A. P., \& Ward-Thompson, D. 2004, A\&A, 423, 169

Güdel, M., Audard, M., Kashyap, V. L., Drake, J. J., \& Guinan, E. F. 2003, ApJ, 582,423

Güdel, M., Briggs, K. R., Arzner, K., et al. 2007, A\&A, 468, 353

Guieu, S., Dougados, C., Monin, J.-L., Magnier, E., \& Martín, E. L. 2006, A\&A, 446, 485

Hunsch, M., Schmitt, J. H. M. M., Schroder, K.-P., \& Zickgraf, F.-J. 1998, A\&A, 330,225

Jacoby, G. H., Hunter, D. A., \& Christian, C. A. 1984, ApJS, 56, 257

Jones, B. F., \& Herbig, G. H. 1979, AJ, 84, 1872

Kenyon, S. J., \& Hartmann, L. 1995, ApJS, 101, 117

Klessen, R. S. 2001, ApJ, 556, 837

Kurucz, R. L. 1970, SAO Special Report, 309

Luhman, K. L. 2004, ApJ, 617, 1216

Luhman, K. L., Briceño, C., Stauffer, J. R., et al. 2003a, ApJ, 590, 348

Luhman, K. L., Stauffer, J. R., Muench, A. A., et al. 2003b, ApJ, 593, 1093

Luhman, K. L., Whitney, B. A., Meade, M. R., et al. 2006, ApJ, 647, 1180

Maggio, A., Vaiana, G. S., Haisch, B. M., et al. 1990, ApJ, 348, 253

Montes, D., \& Martin, E. L. 1998, A\&AS, 128, 485

Muench, A. A., Lada, E. A., Lada, C. J., \& Alves, J. 2002, ApJ, 573, 366

Ozawa, H., Grosso, N., \& Montmerle, T. 2005, A\&A, 429, 963

Padgett, D., Fukagawa, M., Rebull, L., et al. 2006, in BAAS, 38, 947

Padoan, P., \& Nordlund, A. 1999, ApJ, 526, 279

Preibisch, T., Kim, Y.-C., Favata, F., et al. 2005, ApJS, 160, 401

Sánchez-Blázquez, P., Peletier, R. F., Jiménez-Vicente, J., et al. 2006, MNRAS, 371, 703

Scelsi, L., Maggio, A., Micela, G., et al. 2007, A\&A, 468, 405

Siess, L., Dufour, E., \& Forestini, M. 2000, A\&A, 358, 593

Skrutskie, M. F., Cutri, R. M., Stiening, R., et al. 2006, AJ, 131, 1163

Soderblom, D. R., Oey, M. S., Johnson, D. R. H., \& Stone, R. P. S. 1990, AJ, 99, 595

Stelzer, B., \& Neuhäuser, R. 2001, A\&A, 377, 538

Stelzer, B., Flaccomio, E., Briggs, K., et al. 2007, A\&A, 468, 463

Strassmeier, K. G., \& Fekel, F. C. 1990, A\&A, 230, 389

Strom, K. M., Strom, S. E., \& Vrba, F. J. 1976, AJ, 81, 320

Truelove, J. K. 1997, Ph.D. Thesis

White, R. J., \& Basri, G. 2003, ApJ, 582, 1109

Wolk, S. J., Harnden, F. R., Flaccomio, E., et al. 2005, ApJS, 160, 423

Zombeck, M. V. 1991, S\&T, 81, 278

\footnotetext{
4 In this plot, the mass of XEST-11-078 had been estimated under the wrong assumption of spectral type $M$ and no IR excess.
} 